\title{
An interpretation of the Hubble expansion by the antigraviton-graviton theory
}

\author{
Wing-Hong Wong (黃䫋航) *, Wing-To Wong (黃穎濤), Wing-Keung Wong (黃穎強), Ling-Mei Wong (黃靈美) \\ China Evangelical Seminary, Taipei \\ *Corresponding author E-mail: jwhwong@ces.org.tw
}

\begin{abstract}
Building on the foundation of the antigraviton-graviton theory (AGT), which was published recently, in this paper, we shall give a theoretical interpretation of the Hubble expansion of the universe, without adopting the dark matter hypothesis nor the dark energy hypothesis. Our theoretical results may be summarized by two points. Firstly, in contrast to Einstein's general relativity theory (GRT), our AGT predicts that the universe will be expanding, provided that its average density is positive definite. Secondly, our AGT has found that the universe may be divided into two spatial regions. Defining the radius ratio as the ratio of the radius to the radius of the visible universe, it is found that when the radius ratio is less than 0.125 , the expansion of the universe can be nearly exactly described by the Hubble formula. When the radius ratio is between 0.125 and 0.64 , the expansion rate is greater than that given by the Hubble formula. And the theoretical Hubble expansion rate reaches its maximum when the radius ratio is at about 0.64.
\end{abstract}

Keywords: Cosmology: Theory, Dark Energy, Dark Matter, Galaxies: Kinematics and Dynamics, Gravitation, Large-Scale Structure of Universe.

\section{Introduction}

The Hubble expansion of the universe was discovered in 1929 by the astronomer Edwin P. Hubble [1], who found that the recession velocity of a galaxy is linearly proportional to its distance. In other words, the rate at which two galaxies are receding from another one is proportional to their relative distance. For sufficiently distant galaxies, the recession velocities are comparable to that of light.

In reference [2], we have derived an antigraviton-graviton theory (AGT), or a quantum gravity theory (QGT), for explaining the shape of the rotation curve of a typical spiral galaxy. On the basis of reference [2], below we shall give a theoretical explanation of the Hubble expansion of the universe, without using the dark matter hypothesis nor the dark energy hypothesis.

\section{Theory}

\subsection{A model of the visible universe}

Let us assume that macroscopically, the mass distribution of universe is isotropic and uniform. Let the uniform average of density of matter be denoted as $\rho_{\mathrm{O}}$, which is assumed to be equal to the measured average density. In other words, when the radius is large enough, the local anisotropies and non-uniformities of density will average out.

The net gravitational effect of the mass distribution outside the visible universe on the visible universe is zero. Consequently in the following, we need only consider the mass distribution of the visible universe.

\subsection{Gravitational scale-length of the universe}

Let point $\mathrm{O}$ be the centre of the universe, and let the radius of the visible universe be $R_{\mathrm{A}}$. Thus

$R_{\mathrm{A}}=c \tau_{\mathrm{O}}$,

where $c$ is the speed of light, and $\tau_{\mathrm{O}}$ is the age of the universe.

Let us adopt the definition of the radial centre of mass (RCM) as presented in reference [2]. For the universe, let us define the radius of its radial centre of mass (RCM) as the ratio of the first moment about its origin $\mathrm{O}$ to the total mass of the universe, i.e.:

$R_{\mathrm{RCM}}=\int_{0}^{R} \mathrm{~A} 4 \pi \rho_{\mathrm{O}} R^{3} d R / \int_{0}^{R} \mathrm{~A} 4 \pi \rho_{\mathrm{O}} R^{2} d R$,

where $\rho_{\mathrm{O}}$ is the surface density as a function of the radius $\mathrm{R}$. Simplifying, we have

$R_{\mathrm{RCM}}=\int_{0}^{R} \mathrm{~A} \rho_{\mathrm{O}} R^{3} d R / \int_{0}^{R} \mathrm{~A} \rho_{\mathrm{O}} R^{2} d R$.

From eq. (1), it can be shown by integral calculus that

$R_{\mathrm{RCM}}=3 R_{\mathrm{A}} / 4$.

Hence all the mass of the universe may be considered concentrated in an infinitesimal thin spherical shell of radius $R_{\mathrm{RCM}}=3 R_{\mathrm{A}} / 4$. The centrifugal mean free path $\lambda_{\mathrm{O}}$, centrifugal of all the mass in this infinitesimal spherical shell to move outward is equal to the distance between the points at radius $R_{\mathrm{RCM}}$ to the edge of the visible universe, which is equal to $R_{\mathrm{A}}$. Therefore

$\lambda_{\mathrm{O}, \text { centrifugal }}=R_{\mathrm{A}}-R_{\mathrm{RCM}}$ 
Substituting eq. (2) into eq. (3), we obtain

$\lambda_{\mathrm{o}, \text { centrifugal }}=R_{\mathrm{A}} / 4$

Similarly, the centripetal mean free path is

$\lambda_{\mathrm{o}, \text { centripetal }}=R_{\mathrm{A}}-\lambda_{\mathrm{O}, \text { centrifugal }}=3 R_{\mathrm{A}} / 4$

From eq. (4) and eq. (5), the average of $\lambda_{\mathrm{o}}$, centripetal and $\lambda_{\mathrm{o}}$, centrifugal is evaluated to be the average mean free path of the gravitons, which is equal to the fundamental wavelength $\lambda_{\mathrm{o}}$ of the gravitons. Thus,

$\lambda_{\mathrm{O}}=0.5 R_{\mathrm{A}}$

In reference [2], it has been shown that, $R_{\circ}$, the gravitational scale-length of a mass distribution is equal to the fundamental quarter wavelength of the gravitons. Using eq. (6), we have

$R_{\circ}=R_{\mathrm{A}} / 8$

\subsection{The graviton wavelength as a function of the radius in the universe}

In reference [2], we have shown that, $\lambda_{\mathrm{A}}(R)$ the expectation value of the graviton wavelength is given by the following equation

$\lambda_{\mathrm{A}}(R)=R_{\mathrm{O}}\left[1+\frac{\left(R / R_{\mathrm{O}}\right)-1}{1+\ln \left(R / R_{\mathrm{O}}\right)}\right]$

Combining eq. (7) and eq. (8), a graph of $\lambda_{\mathrm{A}}(R)$ is presented in Fig. 1. In this graph, the y-axis is expressed as a dimensionless ratio of the wavelength to $R_{\mathrm{A}}$ (the radius of the universe). Similarly, the $\mathrm{x}$-axis is expressed as a dimensionless ratio of the radius $\mathrm{R}$ to $R_{\mathrm{A}}$. It is a plot of two dimensionless ratios. Qualitatively, the graph shows that the graviton wavelength ratio increases linearly with the radius until the radius is equal to the gravitational scale-length $R_{\circ}$; and then it rises more slowly to a maximum of 0.4091424 ( $7 \mathrm{sig}$. fig.) when the radius ratio is 1.0

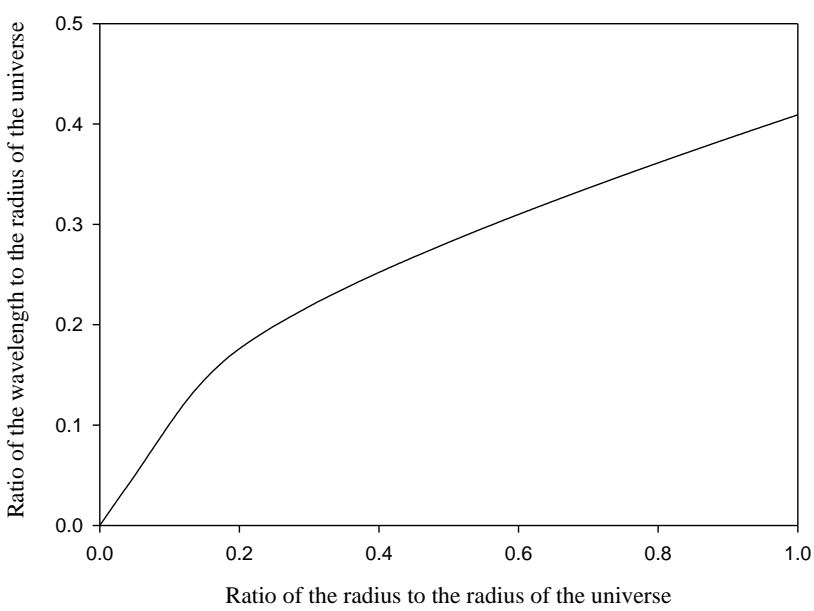

Fig. 1: Graviton Wavelength versus Radius

\subsection{The Hubble speed}

By the principle of energy conservation, the sum of the kinetic energy (K.E.) and the gravitational potential energy (P.E.) of any astronomical object (galaxy, quasar, etc.) is a constant. Let us assume that this constant is zero, we have

K.E. + P.E. $=0$.

Let the mass and the radial speed of an astronomical object be $m$ and $u$ respectively. From eq. (9), we obtain

$\frac{m u^{2}}{2}-m \Phi_{\mathrm{q}}(R)=0$,

where $\Phi_{\mathrm{q}}(R)$ is the quantum gravitational potential. From eq. (10), we obtain

$u^{2}=2 \Phi_{\mathrm{q}}(R)$.

Using the equation for $\Phi_{\mathrm{q}}(R)$, derived by reference [2], we have

$u^{2}=2 \frac{G_{q} M(R) \cosh \left[R / \lambda_{\mathrm{A}}(R)\right]}{R}$,

where $G_{q}$ is quantum gravitational constant, $\mathrm{R}$ is the radial distance of the astronomical object under consideration, $M(R)$ is the central mass at radius $\mathrm{R}$, and $\lambda_{\mathrm{A}}(R)$ is the expectation value of the graviton wavelength respectively.

From eq. (12) and the meaning of $M(R)$, it can be easily shown that

$u^{2}=\frac{8 G_{q} \rho_{\circ} \pi R^{3} \cosh \left[R / \lambda_{\mathrm{A}}(R)\right]}{3 R}$.

From eq. (13), we have

$u=R \sqrt{\frac{8 \pi G_{q} \rho_{\circ} \cosh \left[R / \lambda_{\mathrm{A}}(R)\right]}{3}}$.

If $R \leq R_{\circ}, \lambda_{\mathrm{A}}(R)=R$, and we obtain

$\cosh \left[R / \lambda_{\mathrm{A}}(R)\right]=\cosh (1.0)$

Substituting eq. (15) into eq. (14), we get

$u=H_{\circ} R$,

where $H_{\circ}=\sqrt{\frac{8 \pi G_{q} \rho_{\circ} \cosh (1.0)}{3}}=\sqrt{\frac{8 \pi G_{n} \rho_{\circ}}{3}}$,

and $G_{n}=\cosh (1.0) G_{q}$

It may be noted that eq. (16) is the usual theoretical equation for the Hubble law which was discovered by Edwin Hubble in 1929, but from our AGT, we know that the Hubble law is only applicable in the range of $R \leq R_{\circ}$.

Next, if $R>R_{\circ}$, from eq. (14), 


$$
u=H_{\mathrm{O}} R \sqrt{\frac{\cosh \left[R / \lambda_{\mathrm{A}}(R)\right]}{\cosh (1.0)}} .
$$

Dividing eq. (19) by $c$ the speed of light, we obtain

$$
\beta=\frac{H_{\mathrm{O}} R}{c} \sqrt{\frac{\cosh \left[R / \lambda_{\mathrm{A}}(R)\right]}{\cosh (1.0)}},
$$

where $\beta$ is the ratio of the expansion speed to the speed of light.

$$
\beta=\frac{u}{c} \text {. }
$$

From eq. (16), since the Hubble speed equals the speed of light when the radius is equal to the radius of the visible universe $R_{\mathrm{A}}$, we have

$$
c=H_{\mathrm{o}} R_{\mathrm{A}} \text {. }
$$

Substituting eq. (21) into eq. (19),

$$
\beta=\frac{R}{R_{\mathrm{A}}} \sqrt{\frac{\cosh \left[R / \lambda_{\mathrm{A}}(R)\right]}{\cosh (1.0)}} .
$$

Applying the condition that the $\beta$ ratio is always less than 1.0 , a plot of the $\beta$ ratio $(u / c)$ versus the radius ratio $\left(R / R_{\mathrm{A}}\right)$ is presented in the thick-line curve of Fig. 2. When the radius ratio increases from 0.0 to 0.6407417 ( $7 \mathrm{sig}$. fig.), the $\beta$ ratio increases from 0.0 to 1.0 ; and the slope of the $\beta$ ratio increases otonically. In comparison, a graph of the $\beta$ ratio versus the radius ratio according to Hubble's formula is presented in the dotted line of the same figure. For the Hubble formula, when the radius ratio increases from 0.0 to 1.0 , the $\beta$ ratio increases linearly from 0.0 to 1.0 .

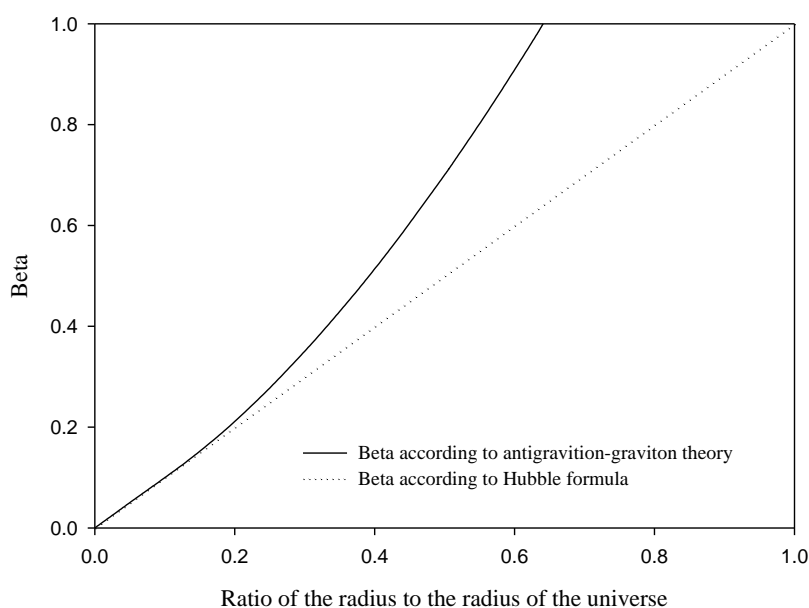

Fig. 2: The Beta Ratio versus the Radius Ratio

\subsection{Relativistic correction}

In eq. (23) of section 2.4, relativistic correction has not yet been applied to the clock rate at radius $\mathrm{R}$. Due to gravitational time dilation, there should be a blue-shift correction factor [3], which is equal to

$\exp \left(-\Phi_{q}(R) / c^{2}\right)$
Substituting eq. (11) into eq. (24), the blue-shift correction factor is

$\exp \left(-u^{2} / 2 c^{2}\right)=\exp \left(-0.5 \beta^{2}\right)$

After applying this blue-shift correction, we obtain the blue-shift corrected beta ratio as

$\beta_{\mathrm{corr}}=\beta \exp \left(-0.5 \beta^{2}\right)$

A plot of the corrected $\beta$ ratio versus the radius ratio is presented in the thick-line graph of Fig. 3. In comparison, a graph of the $\beta$ ratio versus the radius ratio according to Hubble's formula is presented in the dotted line of the same figure. It may be noted that the corrected ratio reaches a maximum value of 0.6065307 ( 7 sig. fig.), when the radius ratio is 0.6407417 . Thus, our AGT predicts that the observed $\beta$ ratio of an astronomical object (such as a galaxy, quasar or supernova) can only reach a maximum value of about 0.6065 ( 4 sig. fig.). This is the prediction of our newly derived relativistic version of AGT. When the radius ratio is between zero to about 0.125 , the corrected $\beta$ ratio is almost equal to the Hubble $\beta$ ratio (e.g. when the radius ratio is 0.125 , the corrected $\beta$ ratio is about $0.7782 \%$ less than that of the Hubble $\beta$ ratio). This may be called the nearly "linear region," where Hubble's formula is nearly exact.

Next, when the ratio is between 0.125 and 0.6407417 (7 sig. fig.), the corrected $\beta$ ratio exceeds the Hubble $\beta$ ratio. This is the "accelerating region." The above is our quantitative theoretical results, and they await empirical comparison, which would require very high $\beta$ ratio or high redshift ( $\mathrm{z}$ ) data.

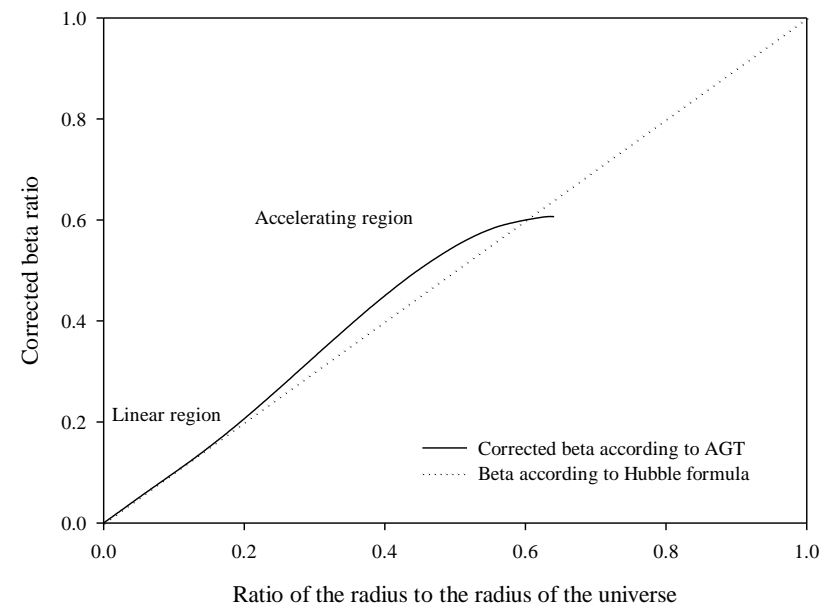

Fig. 3: The Corrected Beat Ratio versus the Radius Ratio

\section{Discussion}

Let us denote the present density of the universe by $\rho_{\mathrm{O}}$ and the present critical density by $\rho_{\mathrm{c}, \mathrm{o}}$ respectively. From Einstein's general relativity theory (GRT), it can be inferred theoretically that there can be three possibilities. Firstly, if $\rho_{\mathrm{O}}$ is larger than $\rho_{\mathrm{c}, \mathrm{o}}$, then the universe will be spatially finite and contracting Secondly, if $\rho_{\mathrm{O}}$ is equal to $\rho_{\mathrm{c}, \mathrm{o}}$, then the universe will be Euclidean and expanding in a linear way, and the Hubble constant is related to the critical density $\rho_{\mathrm{c}, \mathrm{o}}$ by the equation [4] 
$H_{\circ}=\sqrt{\frac{8 \pi G_{n} \rho_{\mathrm{c}, \mathrm{o}}}{3}}$.

Thirdly, if $\rho_{\mathrm{O}}$ is less than $\rho_{\mathrm{c}, \mathrm{o}}$, then the universe will be expanding in an accelerating way.

Among these three possibilities, why is our universe nearly Euclidean and expanding almost in a linear way when the radius is relatively small? Recent astronomical data indicates that when the distance is sufficiently far away, the expansion of our universe is accelerating. The answer given by most astronomers is that for our universe, $\rho_{\mathrm{O}}$ happens to be nearly equal to $\rho_{\mathrm{C}, \mathrm{O}}$. Thus, most astronomers believe that, in the linear region, dark matter exists in our universe, because the amount of luminous matter is insufficient to give us a $\rho_{\mathrm{O}}$ which is nearly equal to $\rho_{\mathrm{c}, \mathrm{o}}$. Moreover, most astronomers or astrophysicists explain the acceleration in the accelerating region of our universe by invoking dark energy also.

\section{Conclusion}

In sum, our theory has derived two notable results.

Firstly, Einstein's general relativity theory (GRT) allows three possibilities as described in sec. 3. In contrast, our quantum gravity theory (QGT) or antigraviton-graviton theory (AGT) predicts that the universe will be expanding, provided that $\rho_{\mathrm{O}}$ is positive definite, see eq. (19) and (23). With due respect to Einstein's GRT, we should realize that it does not know about the existence of the antigravitons, and cannot take into account the cooperation between antigravitons and gravitons in gravitational interactions. Thus it is not so surprising that our result is different from Albert Einstein's GRT.

Secondly, our AGT found that the universe may be divided into two spatial regions. Defining the radius ratio as the ratio of the radius to the radius of the visible universe, it is found that when the radius ratio is less than 0.125 , the expansion of the universe can be almost exactly described by the Hubble formula. This region can be described as the "linear region." When the radius ratio is between 0.125 and 0.6407 ( 4 sig. fig.), the expansion rate is greater than that given by the Hubble formula. This region can be called the "accelerating region."

\section{Acknowledgements}

We are grateful to the anonymous referees for their valuable comments. The encouragement from Wing-Man Wong, Sze-Chun Leung, Chue-Yan Wong and Philip Chi-Wai Wong is hereby gratefully acknowledged.

\section{References}

[1] Hubble E (1929), A Relation between Distance and Radial Velocity among Extra-Galactic Nebulae. Proceedings of the National Academy of Sciences of the United States of America, Vol. 15, No. 3, 168-173

[2] Wong WH, Wong WT, Wong WK \& Wong LM (2014), Discovery of the antigraviton verified by the rotation curve of NGC 6503 . International Journal of Advanced Astronomy, Vol. 2, No.1, 1-7

[3] Rindler W (1977), Essential Relativity. Springer, New York, p.118.

[4] Binney J \& Tremaine S (2008), Galactic Dynamics, $2^{\text {nd }}$ edn. Princeton University Press, Princeton, NJ, p.771. 